\title{
Factorizing Data Technique using Naive Bayes
}

\author{
Rutuja Mane \\ Dept. of Comp. Engg. \\ Sinhagad Institute of Technology \\ Pune, India
}

\author{
A. N. Bandal \\ Dept. of Comp. Engg. \\ Sinhagad Institute of Technology \\ Pune, India
}

\begin{abstract}
Lack of deficiency of information in different particular areas like science, engineering as well as bio informatics has several problems. To overcome these issues, proposed a system and that system fusioning different kind of information inside single or individual unit for the preference or for the research of different existing areas. There is information fusioning is achieved through the matrix factorization based on heterogeneous information datasets that works together upon the proposed system. In proposed system new concept DFMF for the generation of prediction is utilized through the matrix factorization method. Similar system also accomplishes fusion as well as information prediction of the gene and pharmacologic activities.
\end{abstract}

\section{Keywords}

Data fusion, Data integration, Factorization, Bioinformatics, Cheminformatics

\section{INTRODUCTION}

Data fusion methods may combine and works on datasets as well as combine number of data origin at least once. It get problem inside own abstract, discourse as well as craft illustration. Single datasets is additionally not complete, however derivable to its types as well as integral, fusion may improve the strength and prophetical operations of the assuring models.

The most recent branch of information fusion algorithms is mediator (partial) mixture. An algorithm amid this class explicitly faces the assortment of information and consolidates them by deception of single combined model. Middle mixture doesn't coordinate the computer document, nor will it constructed distinctive models for each data provision. It on the other hand holds the pattern of the data sources by including it at intermediate the pattern of prognostic model. This particular methodology is normally most prominent inferable from its superior prognostic precision with the exception of a provided model type, it requires the occasion of a substitution illation principle.

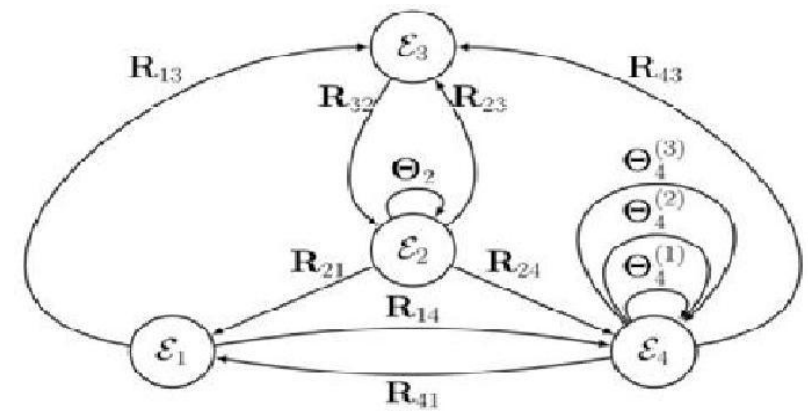

Fig 1: The block-based matrix structure

The report on the occasion of a replacement method for intermediate data integration supported unnatural matrix determining. This system has objective to assembled a rule that requires no or individually smallest transformation of input document and may fuse feature-based representations, affiliations and networks.

\section{LITERATURE REVIEW}

Aerts S, Lamberchts D, Maithy S, proposed [2] the acknowledgment of genes determined in health and sickness remains an issue. This proposed to report a medical science methodology, alongside an openly receptive, intuitive and flexible code termed Endeavor, to extent hopeful genes base biological operations or absconds as well as supported their uniformity to distinguished genes determined in these situations. As compared with previous perceptions, this framework makes unmistakable prioritizations for various diverse data sources that are consolidated or fused within a world ranking misuse order statistics. Moreover, it supplies the flexibility of together with additional data roots. Approval of this framework methodology not opened it completely was prepared to speedily limit 627 genes in sickness information sets and seventy six genes in medical science pathway sets, set up candidates of sixteen mono-or inheritable illnesses and discover regulative tissues of myeloid division.

Jean-Karim Heriche, Jon G. Dregs, Ian Morilla exhibited a paper of the[3] approach of extensive RNA intervention (RNAi) based screens put inside the level to catch genes for all operations human body cells do. Be that as it may, for a few functions, examine gene and value make genome-scale thump down examinations unrealistic. Techniques to predict genes required for cell functions unit therefore needed to concentrate RNAi screens from the whole list over the first conceivable applicants. In spite of the fact that completely distinct medical science tools for element work suspicion exist, they need test validation and area unit along these lines from time to time utilized by analysts. To address this, the framework built up an effective method factor selection system that institute open data viewing genes as graphs so researches these graphs misuse kernels on graph point to consider actual connecting.

Marinka Zitnik and Blaz Zupan propose [4] the average investigation of liver toxicity mixed screening mixes in vivo and in vitro tests. It need to differentiate one from the other between intensifies that shows smallest or no health concern and individuals with the best possibility to happens inverse impacts in people. High-throughput and toxic genomic screening systems not to reference embarrassment of proof offer an issue for improved toxicity consideration and require material machine procedures that incorporate various biological, chemical and pharmacological medicine data. This framework proposed a report on combination approach for supposition of medication-affected liver harm solid in people exploitation microarray information from the Japanese Toxic genomic Project (TGP) as accommodated the competition by CAMDA 2013 Conference. This framework objective was to observe if the information from totally diverse TGP studies may well be mixed along to zest up presumption accuracy.

Brunet JP, Tamayo P, Metagenes portrays here the use of plus 
[5] matrix factoring (NMF), assigned equation supported deterioration by components that may reduce the measurement of expression data from a great many genes to two or three metagenes. Also a model decision operation, hand crafted to figure for any random cluster equation, NMF is assigned temperate technique for perception of different molecular structure and provides a solid procedure for complexity disclosure. This proposed to illustrate the energy of NMF to regain deliberate medical science information from cancer related microarray data. NMF appears to possess advantages over option solutions such various hierarchical or self-sorting out maps. This framework thought that it was less delicate to a priori selection of genes or beginning conditions and ready to locate different or connection subordinate structure of natural phenomenon in convoluted biological frameworks. This capacity, sort of like semantics complexity in content, provides an ordinary technique for solid molecular structure revelation.

\section{IMPLEMENTATION DETAILS}

In this section mentioned the system overview in detail, proposed algorithm, and mathematical model of the proposed system.

\subsection{System Overview}

This paper concentrates upon couple of researches such as bioinformatics as well as cheminformatics. In that recently technological improvement has allows researchers to aggregate as well as separate analyzable datasets. This system proposed for the prediction of genes from bioinformatics and concentrates upon the relation provide via binary matrix relationship inside genes of the amoeba Dictyostelium discoideum as well as all mixed operations or procedures (Gene Ontology (GO)terms). For the pharmacological activities inside the cheminfomatics a subset of chemical for Pub-Chem database this targets binary matrix. In this system, DFMF is utilized to integrate eleven data matrices for gene function prediction and six data matrices for the predictions of pharmocologic activities.

The proposed matrix factorization system demonstrates DFMF novel framework for the data fusioning. Initially this system get inputs the heterogeneous datasets for the combining each datasets into individual component for the pre-processing of the proposed system.

This paper considers six object types such as (Fig.2): genes (type 1), metaphysics operations (type2), observational conditions (type 3), publications from the PubMed info (PMID) (type 4), Medical Subject Headings (MeSH) descriptors (type 5), and KEGG pathways [60] (type 6). The data confined organic phenomenon calculated by completely individual time-points of a 24-hour development cycle [61] (R13 , fourteen experimental conditions), factor annotations with experimental proof code to 148 generic slim terms from the GO (R12), PMIDs and their connected D. discoideum genes from dictyBase(R14), genes taking part in KEGG pathways(R16 ), task of MeSH descriptors to publications from PubMed (R45), references to revealed work on associations between a particular GO term and factor product (R42), and associations of enzymes concerned in KEGG pathways and associated with GO terms(R62 ).

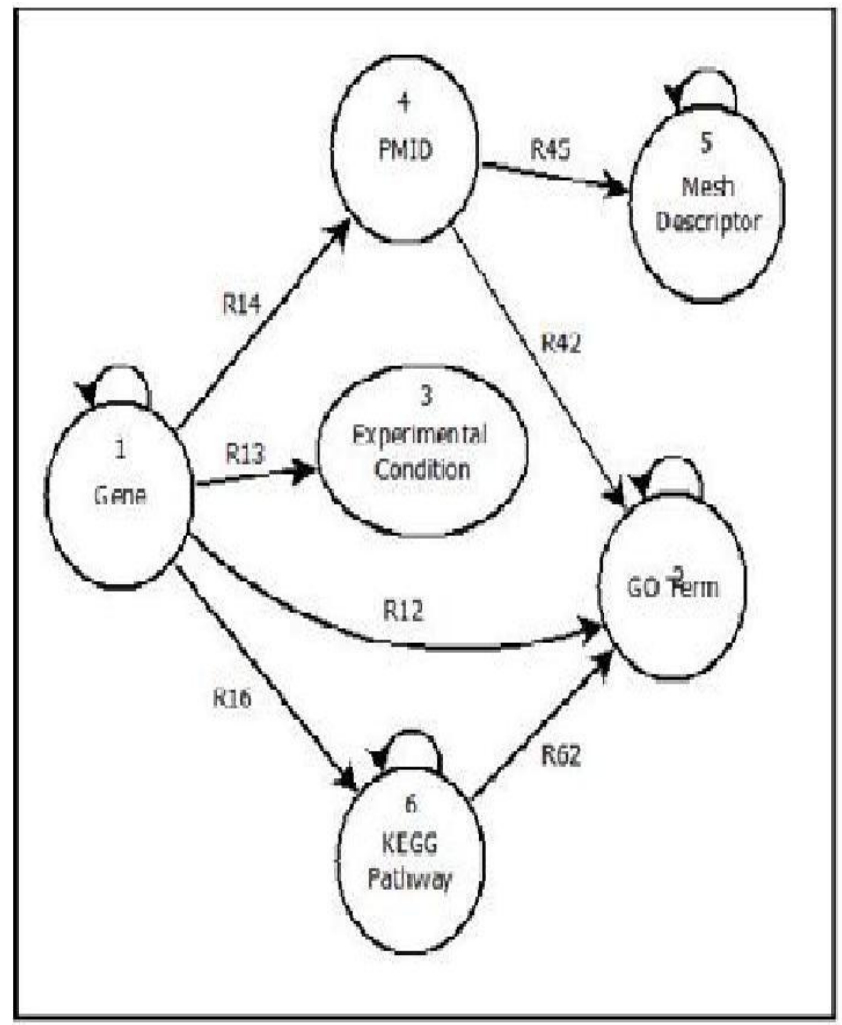

Fig. 2: System Architecture

This system conducted three experiments within which thought of either one hundred or one, most GO-annotated tissues or the fullD. discoideum ordering (12,000 genes). The proposed system presents additionally observe the assumption of sequence integrate with any of $9 \mathrm{GO}$ terms that square measure of particular connection to the present analysis within the Dictyostelium community (upon consultations with $\mathrm{Gad}$ Shaulsky, Baylor faculty of drugs, Houston, TX. rather than employing a generic slim set of terms, this system observe the assumption within the context of an entire set of GO terms. This resulted in very information set with 2,000terms, every term having 10 direct sequence annotations.

\subsection{Kernel Based Fusion}

To demonstrates the information pattern of $\mathrm{MeSH}$ descriptors(Q5 ), the linguistics pattern of the GO graph(Q2 ) and orthodox teams that significant to KEGG pathways (Q6 ), in this system, concept of the genes as points in 3 diverse giant weighted of valued graphs. Inside the graph for Q5, the link inside 2 genes was weighted by the significances of their integrated sets of Mesh descriptors utilize data from R14and $\mathrm{R} 45$. In this system, concept of the MeSH is dividing to live these similarities. At the same time, for the graph for Q2 this system concept of the GO linguistics pattern in operating relevancy of sets of GO terms correlated to genes. Inside the graph for Q6, the cistron edges were weighted by the amount of general KEGGortholog teams. Kernel matrices were generated with a diffusion kernel. 


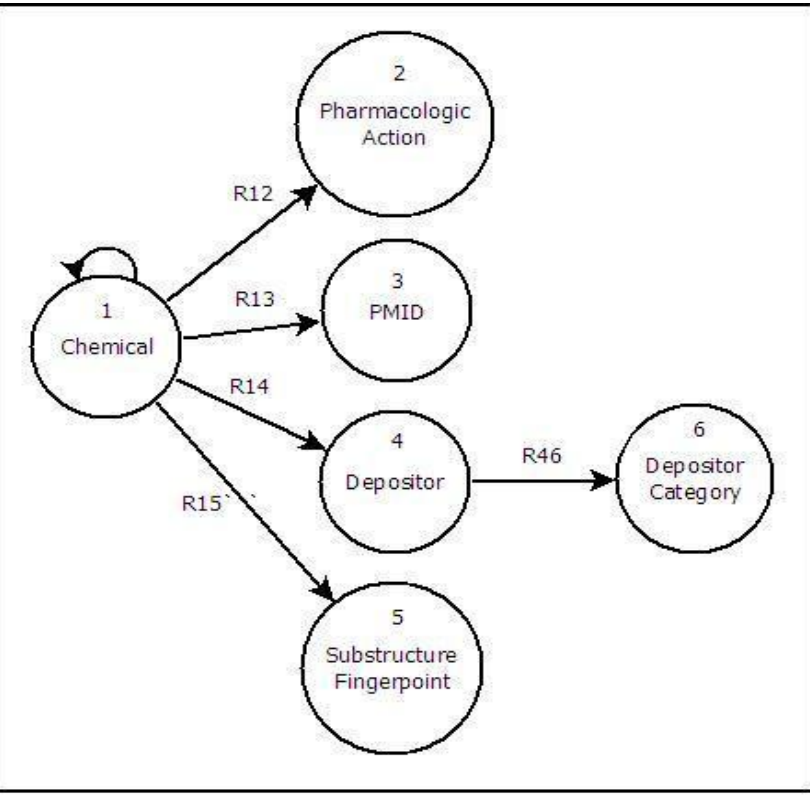

Fig 3: The fusion configuration for the prediction of pharmacologic actions of chemicals

\subsection{Algorithm}

\section{Theorem 1 (Correctness of DFMF algorithm)}

If the update rules for matrix factors $\mathbf{G}$ and $\mathbf{S}$ from Algorithm 1 converge, then final solution satisfies the KKT conditions of optimality.

Input: A set $R$ of relation matrices $R_{i j}$; constraint matrices $\theta^{(t)}$

For $t \in\left\{1,2, \ldots ., \max _{i} t_{i} ; \operatorname{ranks} k_{1}, k_{2}, \ldots . ., k_{r}(i, j \in[r])\right\}$.

Output: Matrix factors S and G.

Initialize $G_{i}$ for $i=1,2, \ldots, r$.

Repeat until convergence:

Construct $\mathrm{R}$ and $\mathrm{G}$ using their definitions using factorization model for multi-relational and multi-object type data.

Update S using:

$$
S \leftarrow\left(G^{T} G\right)^{-1} G^{T} R G\left(G^{T} G\right)^{-1}
$$

Set $G_{i}^{(e)} \leftarrow 0$ for $\{I=1,2, \ldots . r\}$.

Set $G_{i}^{(d)} \leftarrow 0$ for $\{I=1,2, \ldots . r\}$.

For $R_{i j} \in R$ :

$$
\begin{aligned}
& G_{i}^{(e)}+=\left(R_{i j} G_{j} S_{i j}^{T}\right)^{+} G_{i}\left(S_{i j} G_{j}^{T} G_{j} S_{i j}^{T}\right)^{-} \\
& G_{i}^{(d)}+=\left(R_{i j} G_{j} S_{i j}^{T}\right)^{-} G_{i}\left(S_{i j} G_{j}^{T} G_{j} S_{i j}^{T}\right)^{+} \\
& G_{i}^{(e)}+=\left(R_{i j}^{T} G_{j} S_{i j}^{T}\right)^{+} G_{i}\left(S_{i j}^{T} G_{j}^{T} G_{j} S_{i j}^{T}\right)^{-}
\end{aligned}
$$

$$
G_{i}^{(d)}+=\left(R_{i j}^{T} G_{j} S_{i j}^{T}\right)^{-} G_{i}\left(S_{i j}^{T} G_{j}^{T} G_{j} S_{i j}^{T}\right)^{+}
$$

For $t=1,2, \ldots, \max _{i} t_{i}$ :

$G_{i}^{(e)}+=\left[\Theta^{(t) t}\right]^{-} G_{i}$ for $\{i=1,2, \ldots . r\}$

$G_{i}^{(d)}+=\left[\theta^{(t)_{t}}\right]^{-} G_{i}$ for $\{i=1,2, \ldots . r\}$

Construct $\mathrm{G}$ as:

$$
G \leftarrow G_{\mathrm{o}} \operatorname{Diag}\left(\sqrt{\frac{G_{1}^{(e)}}{G_{1}^{(d)}}}, \sqrt{\frac{G_{2}^{(e)}}{G_{2}^{(d)}}}, \ldots ., \sqrt{\frac{G_{r}^{(e)}}{G_{r}^{(d)}}}\right)
$$

Where denotes the Hadamard product. The $\sqrt{ }$ And $\div$ are entry-wise operations.

\subsection{Experimental Setup}

The system is developed using Java framework (version jdk 8) on Windows platform. The Netbeans (version 8.1) is used as a development kit. The system doesn't require any particular hardware to run; any standard machine is able of running the application in any system.

\section{RESULT AND DISCUSSION}

\subsection{Dataset Discussion}

In this system number of datasets is utilized like Gene Ontology (GO), MeSH Discriptor, KEGG pathways etc. These every integrated information worked over the proposed system for information fusion.

\subsection{Results}

The following outcomes demonstrate memory utilization of the system between two systems is as follows:

Table I: Memory Comparison

\begin{tabular}{|l|l|l|l|}
\hline & \multicolumn{3}{|c|}{ Memory in Bytes } \\
\hline Algorithm & 20000 & 40000 & 60000 \\
\hline DFMF & 15255 & 30161 & 46730 \\
\hline MKL & 18579 & 35880 & 52500 \\
\hline
\end{tabular}

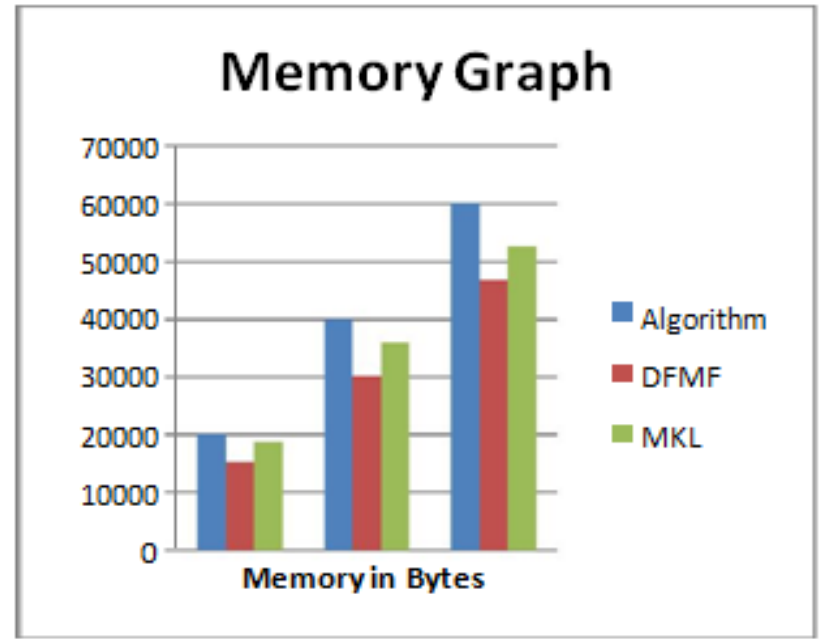

Fig 4: Memory Comparison Graph

The following outcomes demonstrate about gene dataset of two systems. 
Table 2: Memory Comparison between Existing and Propose System

\begin{tabular}{|c|c|c|c|c|}
\hline & \multicolumn{2}{|c|}{ DFMF } & \multicolumn{2}{c|}{ MKL } \\
\hline 100 D.discoideum genes & 0.799 & 0.801 & 0.781 & 0.788 \\
\hline $\begin{array}{c}\text { 1000 D.discoideum } \\
\text { genes }\end{array}$ & 0.826 & 0.823 & 0.787 & 0.798 \\
\hline WoleD.discoideum & 0.831 & 0.849 & 0.8 & 0.821 \\
\hline Pharmacologic actions & 0.663 & 0.834 & 0.639 & 0.811 \\
\hline
\end{tabular}

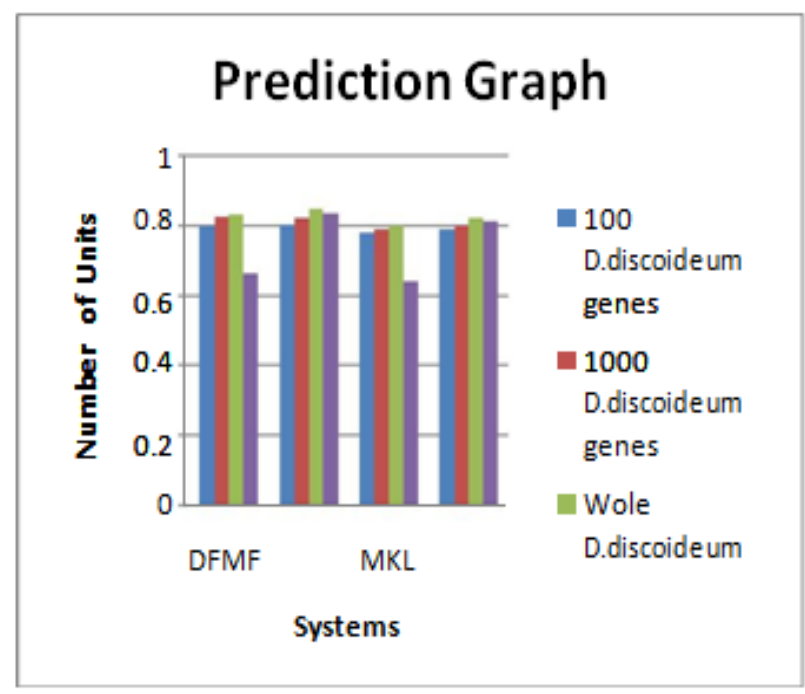

Fig 5: Prediction Graph.

\section{CONCLUSION}

The proposed system performs the fusioning technique through the matrix factorization method by creating several predictions on the basis of the dataset input to the system. That demonstrates the numerous analyses for the specific results. System implements a dataset for the integration of the outcomes for the research as well as analysis of the systems. In this system proposed an algorithm called DFMF for factorization of the all matrix which executes for the appropriate outcomes of the gene as well as another datasets. This system performs classification of all information within a single unit.

\section{REFERENCES}

[1] Marinka Zitnik and Blaz Zupan, Data Fusion by Matrix Factorization, University of Ljubljana, Trzaska 25, SI1000 Ljubljana, Slovenia., 2015.

[2] Aerts S, Lamberchts D, Maithy S, Gene prioritization through genomic data fusion, Laboratory of Neurogenetics, Department of Human Genetics, Flanders Interuniversity Institute for Biotechnology (VIB), University of Leuven, Herestraat 49, bus 602, 3000 Leuven, Belgium, 2006.

[3] Jean-Karim Heriche, Jon G. Lees, Ian Morilla, Integration of biological data by kernels on graph nodes allows prediction of new genes involved in mitotic chromosome condensation, Swiss Institute of Bioinformatics, University of Zurich, 8057 Zurich, Switzerland, 2014.

[4] Marinka Zitnik \& Blaz Zupan, Matrix factorization-based data fusion for drug-induced liver injury prediction, 2014.

[5] 36Brunet JP, Tamayo P, Metagenes and molecular pattern discovery using matrix factorization, The Eli and Edythe L. Broad Institute, Massachusetts Institute of Technology and Harvard University, 320 Charles Street, Cambridge, MA 02141, USA, 2004.

[6] S. M. Rappaport and M. T. Smith, "Environment and disease risks," Science, vol. 330, no. 6003, pp. 460-461, 2010.

[7] S. Aerts, D. Lambrechts, S. Maity, P. Van Loo, B. Coessens, F. DeSmet, L.-C.Tranchevent, B. De Moor, P. Marynen, B. Hassan, P. Carmeliet, and Y. Moreau, "Gene prioritization through genomic data fusion," Nature Biotechnol., vol. 24, no. 5, pp. 537- 544, 2006.

[8] H. Bostrem, S. F. Andler, M. Brohede, R. Johansson, A. Karlsson, J.ovanLaere, L. Niklasson, M. Nilsson, A. Persson, and T. Ziemke, "On the definition of information fusion as a field of research," Univ. Skived, School Humanities Informat, Skovde, Sweden, Tech. Rep. HSIKI-TR-07-006, 2007.

[9] D. Greene and P. Cunningham, "A matrix factorization approach for integrating multiple data views," in Proc. Eur. Conf. Mach. Learn. Knowl. Discovery Databases, 2009, pp. 423-438.

[10] G. R. G. Lanckriet, T. De Bie, N. Cristianini, M. I. Jordan, and W. S.Noble, "A statistical framework for genomic data fusion," Bioin-format., vol. 20 , no. 16 , pp. 2626 2635, 2004.

[11] P. Pavlidis, J. Cai, J. Weston, and W. S. Noble, "Learning gene functional classifications from multiple data types," J. Comput. Biol. vol. 9, pp. 401-411, 2002. 